\title{
On the spacing distribution of the Riemann zeros: corrections to the asymptotic result
}

\author{
E. Bogomolny†, O. Bohigas†, P. Leboeuf $\uparrow$ and A. G. Monastra $\ddagger$ \\ †Laboratoire de Physique Théorique et Modèles Statistiques *, Université de Paris XI, \\ Bât. 100, 91405 Orsay Cedex, France \\ $\ddagger T U$ Dresden Institut für Theoretische Physik, 01062 Dresden, Germany
}

November 19, 2018

\begin{abstract}
It has been conjectured that the statistical properties of zeros of the Riemann zeta function near $z=1 / 2+\mathrm{i} E$ tend, as $E \rightarrow \infty$, to the distribution of eigenvalues of large random matrices from the Unitary Ensemble. At finite $E$ numerical results show that the nearest-neighbour spacing distribution presents deviations with respect to the conjectured asymptotic form. We give here arguments indicating that to leading order these deviations are the same as those of unitary random matrices of finite dimension $N_{\text {eff }}=\log (E / 2 \pi) / \sqrt{12 \Lambda}$, where $\Lambda=1.57314 \ldots$ is a well defined constant.
\end{abstract}

\section{Introduction}

The study of connections between random matrix theory and properties of the Riemann zeta function, $\zeta(z)$, has known recently significant developments [1]. A central point is Hugh Montgomery's (generalized) conjecture [2] that in the asymptotic limit (high on the critical line for $z=1 / 2+\mathrm{i} E)$ the fluctuation properties of non-trivial Riemann zeros are the same as for the circular unitary ensemble $\left(\mathrm{CUE}_{\mathrm{N}}\right)$ of $N \times N$ random unitary matrices (with Haar measure) in the limit of large dimensionality, $N \rightarrow \infty$. In particular, the normalized pair correlation function of the Riemann zeros with $E \rightarrow \infty$ is conjectured to be

$$
R_{2}(s)=1-\left(\frac{\sin \pi s}{\pi s}\right)^{2}
$$

where $s$ is the unfolded distance between zeros (i.e. the mean spacing is set to one). Andrew Odlyzko, since the late 70's, started accurate and extensive numerical computations of Riemann

*Unité de recherche associée au CNRS. 

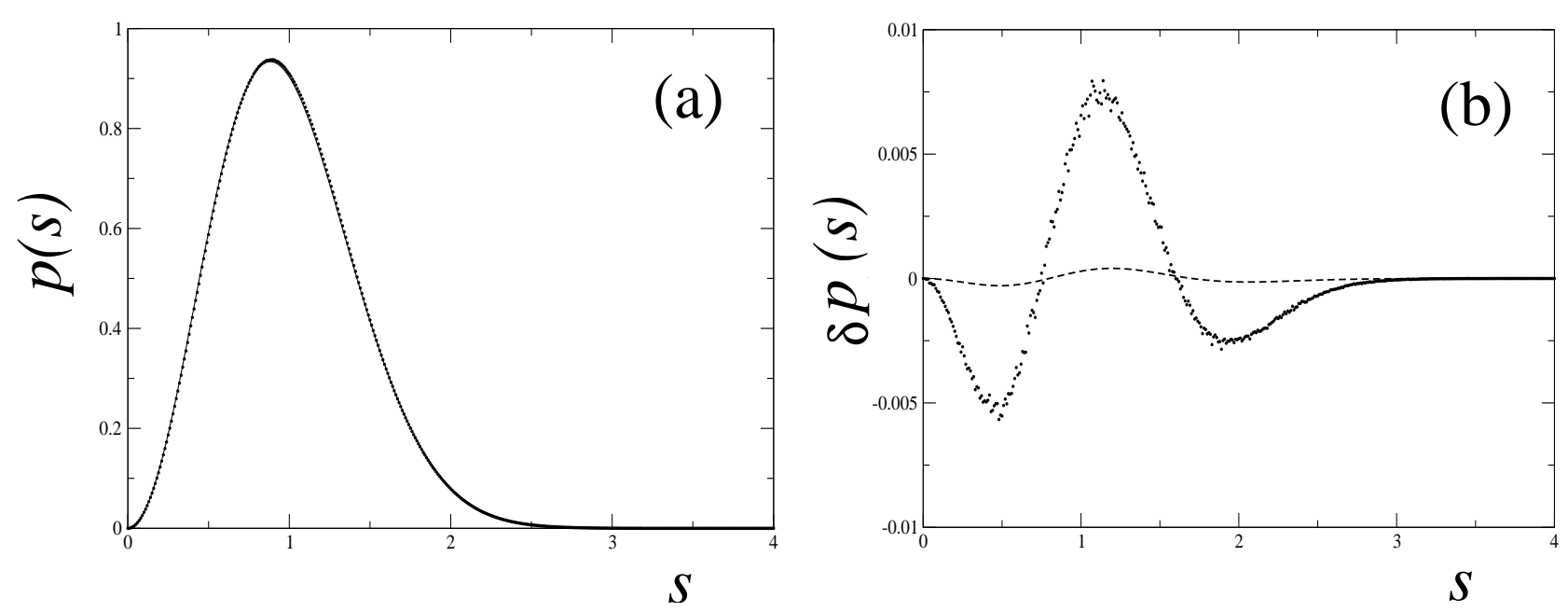

Figure 1: Nearest neighbour spacing distribution of the Riemann zeros located in a window near $E=2.5041178 \times 10^{15}$. (a) Numerical results (dots, from A. Odlyzko) compared to the asymptotic CUE curve (full line), almost indistinguishable. (b) Difference between the numerical result and the asymptotic CUE curve (dots) compared to the difference between the spacing distribution of CUE matrices of size $N_{0}$ (Eq. (2)) and the asymptotic curve (dashed line).

zeros in order to check this and other conjectures [3]. His main result is that in the limit $E \rightarrow \infty$ correlation functions of Riemann zeros do agree with random matrix predictions. For instance, in Fig. 1(a) the density distribution $p(s)$ of spacings among consecutive zeros of $\zeta(z)$ (called the nearest-neighbour spacing distribution in random matrix literature) is plotted for a billion zeros around the $10^{16}$-th zero. The agreement with the CUE asymptotic prediction $p_{0}(s)$ (represented by a solid line in that figure) is remarkable.

It is interesting to look at the difference $\delta p(s)=p(s)-p_{0}(s)$ between the computed and the conjectured distributions (see Fig. 1(b)). Though the difference is small (of order $10^{-2}$ ) it has a clear structure with a nontrivial $s$ dependence.

One may wonder how this difference compares with the one obtained, within random matrix theory, between the asymptotic and a finite $N$ calculation. To proceed, one needs a criterion for the size of the matrix to compare with. The simplest assumption is to choose $N=N_{0}$, where

$$
N_{0}=\log \left(\frac{E}{2 \pi}\right) .
$$

This matrix size is obtained by equating the local density of zeros at height $E$ along the critical line to the density of eigenvalues of the $N \times N$ unitary matrix [4].

This dimensional correspondence has been successful when comparing statistical properties of $\zeta(1 / 2+\mathrm{i} E)$ at finite $E$ with those of characteristic polynomials of $\mathrm{CUE}_{\mathrm{N}}$ matrices of size $N=N_{0}$ [4]. The Riemann zeros in Fig. 1 are located in a window around $E=2.5041178 \times 10^{15}$, which gives $N_{0}=33.6188$. The difference between the finite $N$ (with $N=N_{0}$ ) and the asymptotic nearest neighbour spacing distributions is represented by a dashed line in Fig. 1(b). Though 
the functional form of the correction is qualitatively correct, its amplitude is clearly too small (by a factor of order 20). In his paper [3], A. Odlyzko commented: 'Clearly there is structure in the difference graph, and the challenge is to understand where it comes from'. The purpose of this note is to provide some elements in this direction.

\section{Two-point correlation functions}

Let us first analyze the corrections to the asymptotic two-point function (1) for $N \times N$ random matrices from the circular unitary ensemble $\left(\mathrm{CUE}_{\mathrm{N}}\right)$ and for zeros of the Riemann zeta function. The comparison between them will provide an effective set of rules or correspondences between both sequences.

We start first with random matrix theory. For $N$-dimensional unitary matrices the $n$-point correlation functions are given by [5]

$$
R_{n}^{\left(\mathrm{CUE}_{\mathrm{N}}\right)}\left(x_{1}, \ldots, x_{n}\right)=\left.\operatorname{det}\left(K\left(x_{i}, x_{j}\right)\right)\right|_{i, j=1 \ldots, n}
$$

$K\left(x_{i}, x_{j}\right)$ is the corresponding kernel,

$$
K(x, y)=\frac{\sin (\pi(x-y))}{N \sin (\pi(x-y) / N)}=K_{0}(x-y)+\frac{1}{N^{2}} K_{1}(x-y)+\mathcal{O}\left(N^{-4}\right)
$$

where

$$
K_{0}(s)=\frac{\sin (\pi s)}{\pi s}
$$

and

$$
K_{1}(s)=\frac{\pi s}{6} \sin (\pi s)
$$

In particular, the unfolded two-point correlation function takes the form

$$
R_{2}^{\left(\mathrm{CUE}_{\mathrm{N}}\right)}(s)=1-\left(\frac{\sin (\pi s)}{N \sin (\pi s / N)}\right)^{2}
$$

the expansion (4) leads to

$$
R_{2}^{\left(\mathrm{CUE}_{\mathrm{N}}\right)}(s)=1-\frac{\sin ^{2}(\pi s)}{\pi^{2} s^{2}}-\frac{1}{3 N^{2}} \sin ^{2}(\pi s)-\frac{(\pi s)^{2}}{N^{4}} \sin ^{2}(\pi s)+\mathcal{O}\left(N^{-6}\right) .
$$

This formula expresses the correlation function as the asymptotic result Eq. (1) plus corrections proportional to inverse even powers of the matrix dimension. Notice that the corresponding expansion for random hermitian matrices (Gaussian Unitary Ensemble) is different, it is nonanalytic and includes a term proportional to $N^{-1}$ (cf $\left.[5,6]\right)$.

We now need an equivalent result for the Riemann zeros. An heuristic formula for the twopoint correlation function for these zeros was obtained by Bogomolny and Keating in Ref.[7] using the Hardy-Littlewood conjecture for the distribution of prime pairs (for more details see $[8,9])$. It states that the two-point correlation function of Riemann zeros, $r_{2}(\epsilon)$, is the sum of three terms

$$
r_{2}(\epsilon)=\bar{\rho}^{2}+r_{2}^{(\mathrm{diag})}(\epsilon)+r_{2}^{(\mathrm{off})}(\epsilon)
$$


where the smooth density of zeros, $\bar{\rho}$, is asymptotically

$$
\bar{\rho}=\frac{1}{2 \pi} \log \left(\frac{E}{2 \pi}\right)
$$

and the diagonal, $r_{2}^{(\mathrm{diag})}(\epsilon)$, and off-diagonal, $r_{2}^{(\text {off })}(\epsilon)$, parts are given by the following convergent expressions

$$
r_{2}^{(\operatorname{diag})}(\epsilon)=-\frac{1}{4 \pi^{2}} \frac{\partial^{2}}{\partial \epsilon^{2}}\left[\log |\zeta(1+\mathrm{i} \epsilon)|^{2}+2 \sum_{p} \sum_{r=1}^{\infty} \frac{1-r}{r^{2} p^{r}} \cos (\epsilon r \log p)\right]
$$

and

$$
r_{2}^{(\text {off })}(\epsilon)=\frac{1}{4 \pi^{2}}|\zeta(1+\mathrm{i} \epsilon)|^{2} \mathrm{e}^{\mathrm{i} 2 \pi \bar{\rho} \epsilon} \prod_{p}\left[1-\frac{\left(1-p^{\mathrm{i} \epsilon}\right)^{2}}{(p-1)^{2}}\right]+\text { c.c. . }
$$

Here the summation and the product are taken over all primes $p$. In [9] it was checked that these formulas agree very well with Odlyzko's results for the two-point correlation function of Riemann zeros.

The unfolded two-point correlation function is obtained by measuring distances between zeros in units of the local mean spacing,

$$
R_{2}(s)=\frac{1}{\bar{\rho}^{2}} r_{2}\left(\frac{s}{\bar{\rho}}\right)
$$

We are interested in the corrections to the asymptotic behavior of $R_{2}(s)$ in the limit when $E \rightarrow \infty$. In this limit $\bar{\rho} \rightarrow \infty$ and the argument of $r_{2}$ in Eq.(13) becomes small (keeping $s$ finite). Therefore, one can expand $r_{2}(\epsilon)$ for $\epsilon \ll 1$. To perform the expansion it is convenient to use the known series

$$
\zeta(1+x)=\frac{1}{x}+\sum_{n=0}^{\infty} \frac{(-1)^{n}}{n !} \gamma_{n} x^{n},
$$

where $\gamma_{i}$ are the Stieljes constants, and the following auxiliary expansions:

$$
\frac{\partial^{2}}{\partial \epsilon^{2}} \sum_{p} \sum_{r=1}^{\infty} \frac{1-r}{r^{2} p^{r}} \cos (\epsilon r \log p)=\sum_{n=0}^{\infty} c_{n} \epsilon^{2 n}
$$

where

$$
c_{n}=\frac{(-1)^{n}}{(2 n) !} \sum_{p}(\log p)^{2(n+1)} \sum_{r=1}^{\infty} \frac{(r-1) r^{2 n}}{p^{r}},
$$

and

$$
\prod_{p}\left[1-\frac{\left(1-p^{\mathrm{i} \epsilon}\right)^{2}}{(p-1)^{2}}\right]=1+c_{0} \epsilon^{2}+\mathrm{i} Q \epsilon^{3}+\mathcal{O}\left(\epsilon^{4}\right)
$$

with

$$
Q=\sum_{p} \frac{\log ^{3} p}{(p-1)^{2}} .
$$

Collecting the different terms in the expansion, one gets

$$
r_{2}^{(\mathrm{diag})}(\epsilon)=-\frac{1}{2 \pi^{2} \epsilon^{2}}-\frac{\left(\gamma_{0}^{2}+2 \gamma_{1}+c_{0}\right)}{2 \pi^{2}}+\mathcal{O}\left(\epsilon^{2}\right)
$$


and

$$
r_{2}^{(\text {off })}(\epsilon)=\frac{1}{4 \pi^{2}}\left[\frac{1}{\epsilon^{2}}+\left(\gamma_{0}^{2}+2 \gamma_{1}+c_{0}\right)+\mathrm{i} Q \epsilon+\mathcal{O}\left(\epsilon^{2}\right)\right] \mathrm{e}^{\mathrm{i} 2 \pi \bar{\rho} \epsilon}+\text { c.c. } .
$$

The unfolded two-point correlation function takes therefore the form

$$
R_{2}(s)=1-\frac{\sin ^{2}(\pi s)}{\pi^{2} s^{2}}-\frac{\left(\gamma_{0}^{2}+2 \gamma_{1}+c_{0}\right)}{\pi^{2} \bar{\rho}^{2}} \sin ^{2}(\pi s)-\frac{Q}{2 \pi^{2} \bar{\rho}^{3}} s \sin (2 \pi s)+\mathcal{O}\left(\bar{\rho}^{-4}\right) .
$$

Equation (16) expresses the two-point correlation function of Riemann zeros as the asymptotic random matrix result given by Eq. (1), plus corrections that are proportional to inverse powers of the average density of zeros.

The comparison of Eqs.(8) and (16) shows that the leading terms coincide, as conjectured by Montgomery. To relate sub-leading terms we proceed as follows. Up to $\mathcal{O}\left(\bar{\rho}^{-4}\right)$ in Eq. (16), the term of $\mathcal{O}\left(\bar{\rho}^{-3}\right)$ can be absorbed in the term of $\mathcal{O}\left(\bar{\rho}^{-2}\right)$ by rescaling the $s$ variable. Equation (16) now takes the form

$$
R_{2}(s)=1-\frac{\sin ^{2}(\pi s)}{\pi^{2} s^{2}}-\frac{\left(\gamma_{0}^{2}+2 \gamma_{1}+c_{0}\right)}{\pi^{2} \bar{\rho}^{2}} \sin ^{2}(\pi \alpha s)+\mathcal{O}\left(\bar{\rho}^{-4}\right)
$$

where

$$
\alpha=1+\frac{Q}{2 \pi \bar{\rho}\left(\gamma_{0}^{2}+2 \gamma_{1}+c_{0}\right)}=1+\frac{C}{\log (E / 2 \pi)},
$$

$C=Q /\left(\gamma_{0}^{2}+2 \gamma_{1}+c_{0}\right)=1.4720 \ldots$ The comparison of Eqs. (8) and (17) leads to the following conclusions:

i) To leading order in $1 / \bar{\rho}$ the two-point correlation function of the Riemann zeros coincides with that of eigenvalues of random $\mathrm{CUE}_{\mathrm{N}}$ matrices of effective dimension $N=N_{\text {eff }}$, where

$$
N_{\text {eff }}=\frac{\pi \bar{\rho}}{\sqrt{3 \Lambda}}=\frac{1}{\sqrt{12 \Lambda}} \log \left(\frac{E}{2 \pi}\right)
$$

where $\Lambda \equiv \gamma_{0}^{2}+2 \gamma_{1}+c_{0}=1.57314 \ldots$

ii) The next-to-leading order is obtained by rescaling the variable $s$ in the first correction term according to

$$
s \rightarrow \alpha s
$$

where $\alpha$ is given by Eq.(18).

The effective matrix size obtained from our analysis is, therefore, different from $N_{0}$ (Eq. (2)), the relation between them being a multiplicative factor $N_{\text {eff }}=(12 \Lambda)^{-1 / 2} N_{0}=0.230158 N_{0}$.

\section{The nearest-neighbour spacing distribution}

In general, to compute the nearest-neighbour spacing distribution it is necessary to know correlation functions of arbitrary number of points. Though for the Riemann zeros the HardyLittlewood conjecture is sufficient to obtain all correlation functions [10], the computations are cumbersome and for simplicity we shall use another method. 

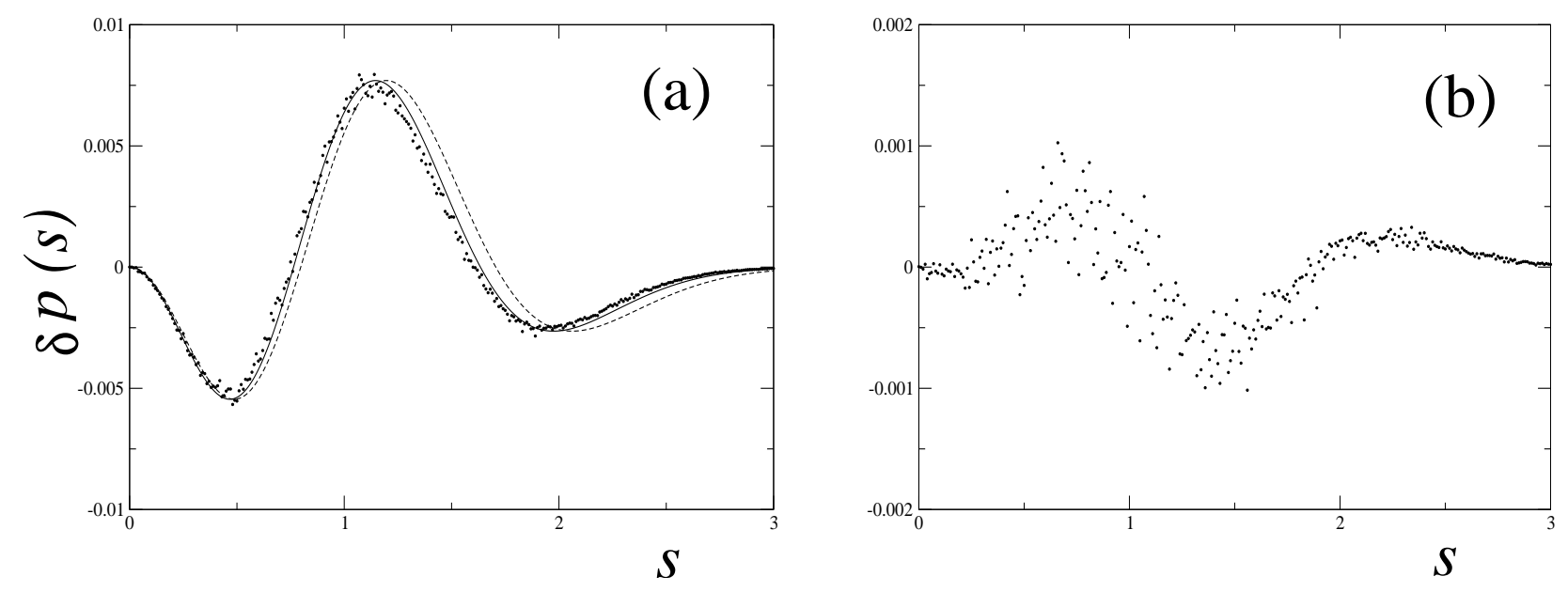

Figure 2: (a) Difference between the nearest neighbour spacing distribution of the Riemann zeros and the asymptotic CUE distribution for a billion zeros located in a window near $E=2.5041178 \times 10^{15}$ (dots), compared to the theoretical prediction Eq.(24) (full line). The dashed line does not include the scaling of $s$. (b) Difference between the numerical values for the Riemann zeros (dots) and the full curve (theory) of part (a).

For CUE, the nearest-neighbour spacing distribution may be expressed as [5]

$$
p^{\left(\mathrm{CUE}_{\mathrm{N}}\right)}(s)=\frac{d^{2} E(s)}{d s^{2}}
$$

where

$$
E(s)=\operatorname{det}\left[\delta_{j k}-\frac{\sin \left(\frac{\pi s}{N}(j-k)\right)}{\pi(j-k)}\right], 1 \leq j, k \leq N .
$$

Some physical arguments [11] indicate that to leading order, under quite general conditions deviations from standard random matrix theory in systems with no time reversal symmetry are reduced to a change of the kernel only, and that the correction term is the same as for $\mathrm{CUE}_{\mathrm{N}}$ matrices with an effective matrix size.

If we accept this conjecture then from the results of the previous section it follows that to leading order all correlation functions of the Riemann zeros are the same as those of $\mathrm{CUE}_{\mathrm{N}}$ matrices with effective dimension given by Eq. (19). In particular, it means that the nearestneighbour spacing distribution of the Riemann zeros can be calculated as follows. First, find the expansion of the nearest-neighbour distribution for $\mathrm{CUE}_{\mathrm{N}}$ random unitary matrices in inverse powers of $N$, namely

$$
p^{\left(\mathrm{CUE}_{\mathrm{N}}\right)}(s)=p_{0}(s)+\frac{1}{N^{2}} p_{1}(s)+\mathcal{O}\left(N^{-4}\right) .
$$

(The fact that the first correction is of order $N^{-2}$ follows from results in [6]). The expansion (23) is difficult to derive analytically. We have therefore computed numerically the spacing distribution $p^{\left(\mathrm{CUE}_{\mathrm{N}}\right)}(s)$ from Eqs. (21-22). The correction term is then obtained by computing 

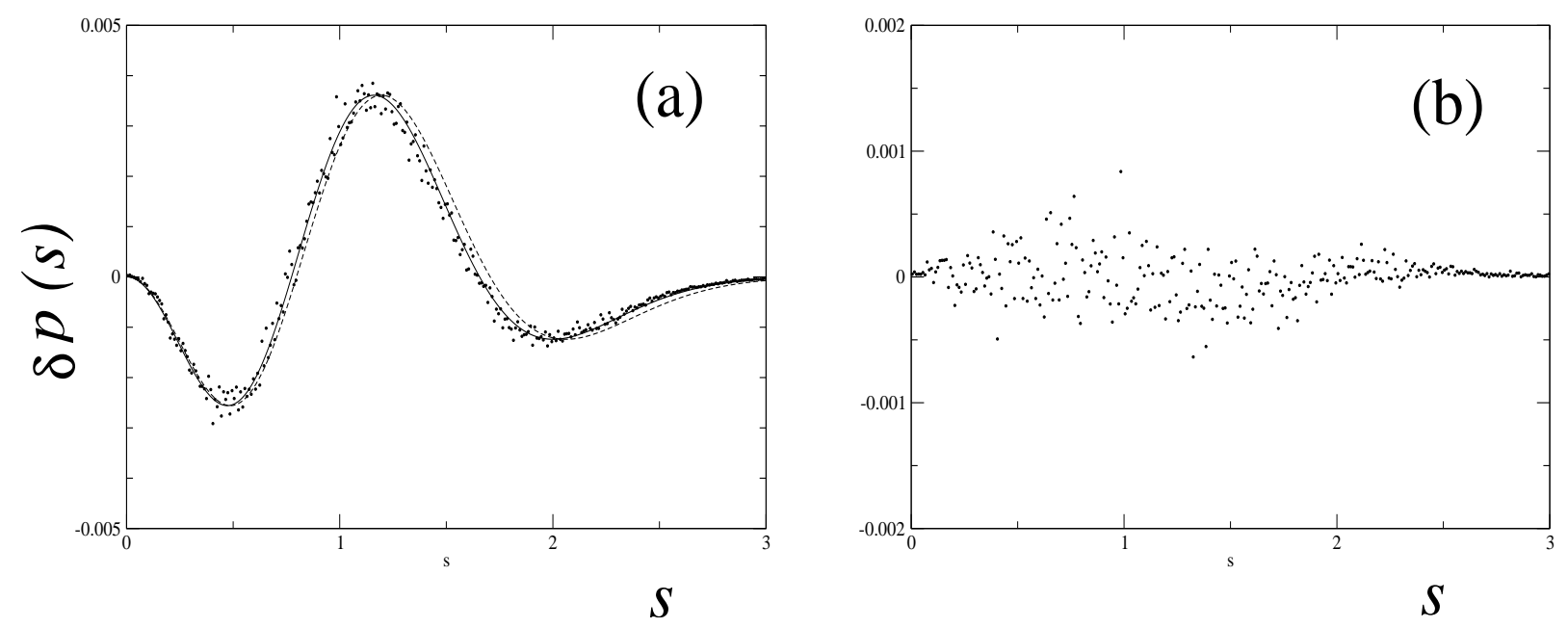

Figure 3: Same as in Fig. 2 but for a billion zeros located in a window near $E=1.30664344 \times 10^{22}$.

$p_{1}(s)=N^{2}\left[p^{\left(\mathrm{CUE}_{\mathrm{N}}\right)}(s)-p_{0}(s)\right]$ for increasing values of $N$. An alternative method would be to use a non-linear differential equation for $p^{\left(\mathrm{CUE}_{\mathrm{N}}\right)}(s)$ as derived in [6].

Second, to leading order, at a given height $E$ above the real axis, replace in Eq. (23) $N$ by $N_{\text {eff }}$ given by Eq. (19). Finally, we shall approximate the next-to-leading correction of $p(s)$ according to the rule (20). In such an approximation the nearest-neighbour spacing distribution of the Riemann zeros equals the universal random matrix result, $p_{0}(s)$, plus the correction $\delta p(s)$, where

$$
\delta p(s)=\frac{1}{N_{\mathrm{eff}}^{2}} p_{1}(\alpha s)+\mathcal{O}\left(N_{\mathrm{eff}}^{-4}\right) .
$$

$N_{\text {eff }}$ and $\alpha$ are given by Eq. (19) and (18), respectively.

Figure 2(a) shows the comparison between the numerical results and Eq.(24) for zeros located on a window around $E=2.5041178 \times 10^{15}$ (as in Fig. 1). The effective matrix size is $N_{\text {eff }}=$ 7.7376 (instead of $N_{0}=33.6188$ ), and $\alpha=1.0438$. The agreement is quite good, and shows that $N_{\text {eff }}$ is the correct matrix size in this case. For comparison, we have plotted as a dashed curve the theoretical formula (24) without the rescaling of the variable $s$.

Figure 2(b) is a plot of the difference between Odlyzko's results and the prediction (24). There is still some structure visible, which might be attributed to the $\mathcal{O}\left(N_{\text {eff }}^{-4}\right)$ correction. To test the convergence, we have made the same plot but now using one billion zeros located on a window around $E=1.30664344 \times 10^{22}$, which corresponds to $N_{\text {eff }}=11.2976\left(\right.$ instead of $\left.N_{0}=49.0864\right)$ and $\alpha=1.0300$ (Fig. 3(a)). Now the agreement is clearly improved. The difference between the prediction (24) and the numerical results, plotted in Fig. 3(b), shows a structureless remain.

\section{Conclusion}

For zeros of the Riemann zeta function located around $z=1 / 2+\mathrm{i} E$, we derived an heuristic formula for the nearest-neighbour spacing distribution that contains finite $-E$ corrections. We 
argued that to leading order the corrections are the same as for random matrices from the circular unitary ensemble of size $N_{\text {eff }} \approx 0.230158 \log (E / 2 \pi)$. We proposed to describe the next to leading order correction as a simple scaling of the dominant term.

Two main conjectures were used. The most important is the explicit expression for the two-point correlation function for the Riemann zeros (Eqs. (11) and (12)) obtained in [7]. The second is the statement that to leading order deviations from random matrix predictions reduce to a change of the kernel (4). It follows that our procedure to compute the finite- $E$ corrections of the complex Riemann zeros from unitary matrices should be valid for any local statistics.

It is known that at finite heights $E$ above the real axis the appropriate symmetry group of the Riemann zeta function is not $\mathrm{CUE}_{\mathrm{N}}$. It has been conjectured [12] that $\zeta(z)$ is a member of a family obeying the symplectic symmetry $U S p(2 N)$. The possible connections of our results with finite $-E$ corrections associated to this symmetry deserve to be investigated.

We acknowledge discussions and data from A. Odlyzko.

\section{References}

[1] P. J. Forrester, N. C. Snaith and J. J. M. Verbaarschot, eds, Special Issue: Random Matrix Theory, J. Phys A 36 (2003) 2859-3646; F. Mezzadri, N. C. Snaith, eds, Recent Perspectives in Random Matrix Theory and Number Theory, Cambridge University Press, Cambridge, 2005.

[2] H. L. Montgomery, The pair correlation of zeros of the zeta function, in Analytic Number Theory 24 (1973) 181-193.

[3] A. M. Odlyzko, The $10^{22}$-nd zero of the Riemann zeta function, in Dynamical, Spectral, and Arithmetic Zeta Functions, M. van Frankenhuysen and M. L. Lapidus, eds., Amer. Math. Soc., Contemporary Math. series 290 (2001) 139-144.

[4] J. P. Keating and N. Snaith, Random matrix theory and $\zeta(1 / 2+\mathrm{i} t)$, Comm. Math. Phys. 214 (2000) 57-89.

[5] M. L. Mehta, Random Matrices, 2nd ed., Academic Press, New York, 1991.

[6] C. A. Tracy and H. Widom, Fredholm determinants, differential equations and matrix models, Commun. Math. Phys. 179 (1996) 1-9.

[7] E. B. Bogomolny and J. P. Keating, Gutzwiller's Trace Formula and Spectral Statistics: Beyond the Diagonal Approximation, Phys. Rev. Lett. 77 (1996) 1472-1475.

[8] E. B. Bogomolny, Spectral statistics and periodic orbits, in New Directions in Quantum Chaos, Proceedings of the International School of Physics "Enrico Fermi", course CXLIII, G. Casati, I. Guarneri and U. Smilansky, eds, Varenna, 2000, 333-368. 
[9] E. Bogomolny, Quantum and Arithmetical Chaos, in Frontiers in Number Theory, Physics and Geometry, Proceedings of Les Houches winter school 2003, Springer-Verlag, 2006.

[10] E. B. Bogomolny and J. P. Keating, Random matrix theory and the Riemann zeros I: three-and four-correlations, Nonlinearity 8 (1995) 1115-1131, ibid II: n-point correlations, Nonlinearity 9 (1995) 911-935.

[11] A. V. Andreev and B. L. Altshuler, Spectral statistics beyond random matrix theory, Phys. Rev. Lett. 75 (1995) 902-905; S. M. Nishigaki, Level spacings at the metal-insulator transition, Phys. Rev. E 59 (1999) 2853-2862.

[12] N. M. Katz and P. Sarnak, Zeros of zeta functions and symmetry, Bull. Am. Math. Soc. 36 (1999) 1-26. 